\title{
USING A MULTI-AGENT SYSTEM FOR SUPPLY CHAIN MANAGEMENT
}

\author{
L. C. M. PERERA \& A. S. KARUNANANDA \\ Department of Computational Mathematics, University of Moratuwa, Sri Lanka.
}

\section{ABSTRACT}

Supply chain management (SCM) is a well-known example of a complex system. Classical computing technologies have shown little success in modeling complex systems. However, a large body of research in multi-agent system (MAS) technology has demonstrated how complex systems can be modeled to generate smart solutions, which could not be done otherwise. We have researched on the design and development of MAS for SCM. In this solution, each phase in the supply chain has been developed as an agent enabling communication, coordination and negotiation among the agents to achieve intended business goals. The study investigated decentralized collaborative planning architecture and agents are attached to different containers of the system. The containers have been implemented using a Java Agent Development Framework (JADE) and consist of diverse methods to support collaboration in the supply chain environment. Agents have different behaviors and their decisions are based on defined ontology. The identified key roles in the supply chain are raw material suppliers, manufacturers, distributors and retailers. They perform autonomous tasks with collaboration to accomplish final customer satisfaction.

Keywords: multi-agent system, supply chain management.

\section{INTRODUCTION}

Complexity in communication in supply chain management (SCM) has been cited by many researchers [2]. In general, the entities operating in a supply chain environment have been provided with limited opportunities for information sharing and collaborative planning. Undisputedly, identification of different entities in a business process and working together to achieve intended goals for an organization is an essential requirement. SCM consists of material venders, suppliers, manufacturers, distributors, retailers and customers to work as network of agents to achieve a common goal. There are some developed legacy systems and client server architecture applications that have provided marginal benefit for the effective communication in SCM. Although there are several automated solutions for SCM, which use classical software technologies but the issues remain unsolved. However, a large volume of literature has shown the potential of multi-agent system (MAS) technology to effectively use the power of communication as a problem-solving strategy in complex systems.

Multi-agent technology has been used in many areas but industry applications have taken the earliest advancement of agent technology when compared to others. The features of MAS technology can be simply used to handle the complexity in communication, which plays the vital role in SCM. OASIS is an air traffic control system used in Sydney airport, Australia can be introduced as an example for efficient usage of MAS in communication [1]. It consists of agents and they are created automatically when aircrafts arrive at the Sydney airport. Their behavior is goal directed and reactive to capture real-time constraint into accounts.

Collaborative planning architecture is an inherent solution for effective communication [2]. It improves the accuracy in the decision-making process and also established a link between internal and external entities to increase the performance in SCM. This paper consists of multi-agent-based solution to eliminate communication complexity in supply chain environment. 
The rest of the paper is organized as follows. Section 2 presents aim and objectives. Section 3 discusses the current trends and issues of SCM while Section 4 describes multi-agent technology. Section 5 explains our approach to SCM using MAS technology. Section 6 deals with design of SCM followed by discussion on our proposed methodology in section 7. Results of the effective communication are dealt with in section 8 . Section 9 describes conclusion and future work.

\section{AIM AND OBJECTIVES}

Our aim is to critically study the SCM domain with a view to identify current practices and issues. Analyze the existing solution in SCM with the view to define the research problem and possible technology. Complete depth study about multi-agent technology and its applications to design and implement MAS solution for SCM. Finally, evaluate the MAS using a real-world scenario.

\section{CURRENT TRENDS AND ISSUES IN SCM}

There are legacy systems that have been developed for SCM [2]. Such systems include advanced planning systems (APS), enterprise resources planning (ERP) and e-commerce systems. Supply chain can be viewed as connected ultimate suppliers and ultimate customers. The suppliers distribute merchandise at the right quantities to the right locations at the right time to minimize the systemwise cost, while satisfying the service-level requirements. In SCM, performance depends on willingness and coordination with others. As a result of this one entity in supply chain is necessarily affecting the performance of the other entity. However, suppliers are inclined to make own decisions in SCM. They involve in many organizations but, sometimes have to depend on decisions made by manufacturers [3].

Effective communication and information sharing improve working capital by $25 \%$ [7]. Regardless of the business type in the SCM process, the primary objective of the every business entity is to earn maximum profits by increasing working capital, streamlining accounts, managing debtors and eliminating unnecessary cost. Therefore, the importance of accurate communication mechanism is an essential factor for the SCM. The introduction of agent technology and Java Agent Development Framework (JADE) platform has fulfilled the needs of effective communication mechanism. SCM is regarded as a strategic essential factor in obtaining great deal of competitiveness in the business environment [8]. In SCM if there is one single company exists in one directional way collaboration may not be useful and not complicated. However, cumulative business growth and connection with other companies in the business process agent technology can be introduced as promising candidate in SCM. SCM, network has facilitated different functions of distribution options for procumbent materials, convert it to intermediate and finished goods and finally distribute over-the-end customers [9].

According to the swarm intelligence, collections of agents work like ants or honey bees [10]. Their searching starts from parallel points and each member carries out intended operation to maximize utility function for sharing information with others. As a new technology paradigm for conceptualizing, designing and implementing computer applications and to eliminate potential issues related to current software applications was achieved using agent technology [11]. SCM is a solution to the some industrial issues [1]. Therefore, the bullwhip effect [12] can be identified as a major problem in the domain of SCM. In SCM, a single modification has done by the end customer and it affects to the entire process is named as bullwhip effect. From the industry viewpoint, production planning, inventory management and transport are the main areas of finding significant problems in SCM. However, handling these entities as an individual and to manage them to behave using their own decisions is rather complicated and difficult. For instance, transport and the inventory management are known as N-P hard problems and are very difficult to handle because all of these entities 
are interdependent. In order to eliminate above constraints needs a system, which supports collaborative planning and information sharing. Object modeling and simulation are new mechanisms that have been developed for SCM [13]. Object modeling have facilitated modeling and individual agents. However, throughout their implementation have not indicated the use of ontologies, message parsing techniques and collaboration for effective communication.

Procedural reasoning system architecture is one of the best architecture of multi-agent technology [14]. It has been deployed in many industry applications not only in SCM but also in other complex commutation systems. However, as a simplest architecture for an agent can be introduced as belief, desire and intention architecture also called as BDI architecture [15] which consists of simplest characteristics, associated for intelligence agents. Supply chain is denoted as complex dynamic system and every member within the system engage the local interactions [16]. MAS have permitted satisfactory results for problems with higher complexity by using classical mathematical model [17]. Development of software agents for distributed data processing can be accomplished by using a framework [18]. They also discussed that growth of contact centers, e-commerce's and value chain reside additional problems of entropies data management and exploitation.

Layered MAS architecture is based on agent commitments [19]. It has illustrated that agents are as components and their interconnections specified as a term of commitments. Combine architecture is an apparent solution for eliminate communication issues in SCM [20]. Today's most of the supply chain functions are static and relay on long-term relationships among trading partners but, more dynamic and flexible practices between entities in SCM provide better solution for customers and suppliers [21]. Introduction of theoretical framework model for collaborative inventory management has effectively benefited successfulness of the SCM [22]. However, the intended results were limited to conceptual and theoretical implementation. To optimize supply chain performance need effective coordination among the entities but due to dynamics of the business operation in an organizational environments are facing difficulties in all aspects. Therefore, sometimes it leads to delayed shipments, production failures, employees are informed as sick and customers change their requirements [23]. In real-world business environments, there are no obligations for any organizations to remain with the supply chain. According to their own judgment, companies can join or leave at any time from the SCM process [24]. In particular, our solutions have implemented using JADE environment and included supply chain functions in real time with more customer involvements for the purpose of wastage reduction, increase overall profits margins and finally to satisfy the customer requirements.

In view of the above, we identify the research problem as the lack of proper coordination and communications in supply chain environment have resulted in malfunctioning of whole process and leading to customer dissatisfaction, profit loss and increase wastage.

\section{MULTI-AGENT TECHNOLOGY}

The concept of MASs has become imperative in artificial intelligence and computer science due to the power of its features and wide industry acceptability. The theory behind agent is, question about what is an agent and the use of mathematical formalisms for representing and reasoning the properties of an agents [25]. However, MAS technology is combination of group of agents sharing information and acting collaboratively to achieve common goals. There are many software projects that have been used in MAS technology to eliminate communication complexity in various domains, including SCM in distributed environment. An agent technology has shown great potential for solving complex distributed problems in over last decades with the growth of inherent distribution. Therefore, decomposition of large-scale systems to individual agents has achieved their common goals by interaction with others [26]. 


\subsection{Features of agents}

In a broader sense, agents could be a kind of software or hardware working on behalf of someone with some specific features [27]. Among others, agents are autonomous so that they can operate without the direct involvement of humans and others and retaining some kind of control of their actions and internal state. Agents can also interact with other agent (sometimes humans) via some kind of agent communication language (ACL). The ACL [28] has been proposed by the Foundation for Intelligent Physical Agents as a proposed standard language for agent communications. Knowledge Query and Manipulation Language is another proposed standard [6, 29]. Agents can perceive the environment changes and can respond in a timely manner what has occurred on it. It may be from physical world, change in the graphical user interface, collection of other agents, from Internet and sometimes combination of all. They simply not act in response to the environment but, able to exhibit the goal-directed behavior by taking the initiatives.

\section{OUR APPROACH TO SCM USING MAS TECHNOLOGY}

This section presents our approach by describing the hypothesis, inputs, outputs, processes, features and users of the novel solution. The proposed solution will be able to model complex system for SCM to address the issues of communication among the parties involved in the process [29].

MAS for SCM have been designed to accept multiple inputs coming from different entities of the supply chain process. There are two modes of output generated by the system. These outputs are coming as organizational-related and the customer-related aspects. Having entered the inputs to the system uses MAS technology and ontology to generate the outputs. For the purpose of understanding, we have illustrated the inputs and outputs generated from raw material supplier agents and manufacturer agents. Raw materials, demand from the manufacturer agents, capacity bookings (man, machine and space) are the inputs from the raw material agents while supplied quantities, customer details, delivery schedules, material cost and future forecasting are generated as outputs. The customer demand is primary input of the manufacture agent. However, capacity bookings, delivery schedules and raw material prices and supplier details are the other inputs for the manufacturer agents. Whereas invoices, dispatch details, quality reports and delivery schedules are the generated outputs from the manufacture agents.

The knowledge required for agents to operate is stored in a common domain ontology and personal ontology. Agents basically access their personal ontologies to generate decisions. Our solution has separate agents for selecting suppliers and generates decisions about pricing, discounts, quality of the products, after service and the past records of entities in SCM. However, agents access common domain ontology that has broad description of the entire supply chain process and made available to any agent with define permissions. Inventory management rules are introduced such as FIFO (First in First Out), LIFO (Last in First Out) according to the product category and availability of stocks.

As a promising technology JADE [5] has been used to automate entire SCM process and Eclipse Luna has used as development environment. Overall features of the proposed system are not derived from the inputs and outputs and are basically non-functional requirements of the system. Agents are created using framework therefore each agent is attached to the containers consumes limited resources and produce high performance. Despite of other multi-agent technologies JADE is available as free software component hence development cost is marginal. Ability to perform under limited resource environment, installation and access through the mobile devises increased the rapid growth of multi-agent technology. Instead of the standalone environment the agent is accessible through the web interface with minimum bandwidth. 


\section{DESIGN OF MAS-BASED SCM}

Previous section has described the approach to the SCM, including hypothesis, inputs, outputs, process and features of the system. In this section, the main objective is to present a high-level understanding of the design architecture, modules and relationships among the different entities involved in SCM.

\subsection{Two components architecture of SCM}

In SCM, information sharing and collaborative planning are the most principal features have been used to eliminate complexity in distributed communication. Therefore, it can be introduced as parallel components and are highly coupled each other in communication.

\subsection{Entities involve in SCM}

There are four main categories of entities (modules) that have been identified namely raw material suppliers, manufacturers, distributors and retail suppliers. All of these entities access common ontology and their personal ontologies while information sharing and collaborative planning. They are sometimes found as hierarchical within the supply chain process but in most cases they have a parallel behavior.

Figure 1 illustrates who are the responsible entities involved in the SCM process and their dependencies. According to Fig. 1, there are limited number of manufacturer agents that operate in the process and they hold significant responsibility when compared to raw material suppliers. In other words, they have more control over the entire supply chain process. However, retail supplier agents are dealing with the customers and any bullwhip effect from the customers end will lead to substantial change in whole SCM process. Therefore, handling the behaviors of each entity is a demanding tasks, and essential to implement comprehensive knowledge base including domain and personal for smooth functioning of the SCM process.

\subsection{Relationship among the entities in SCM}

Figure 2 describes that agent behaviors, roles and responsibilities. Each agent is attached to different containers and is authenticated by main container of the system. The framework we have used to implement containers is described in detail in implementation section [30,31].

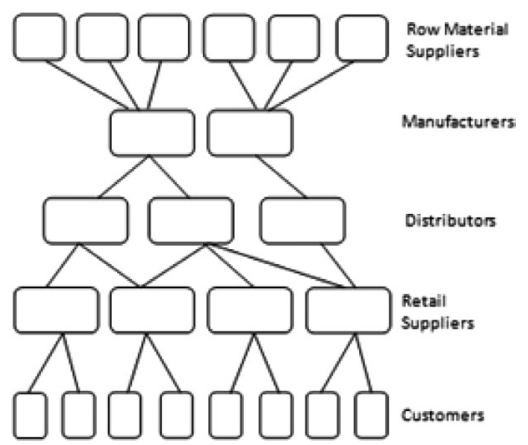

Figure 1: Module-based SCM architecture. 


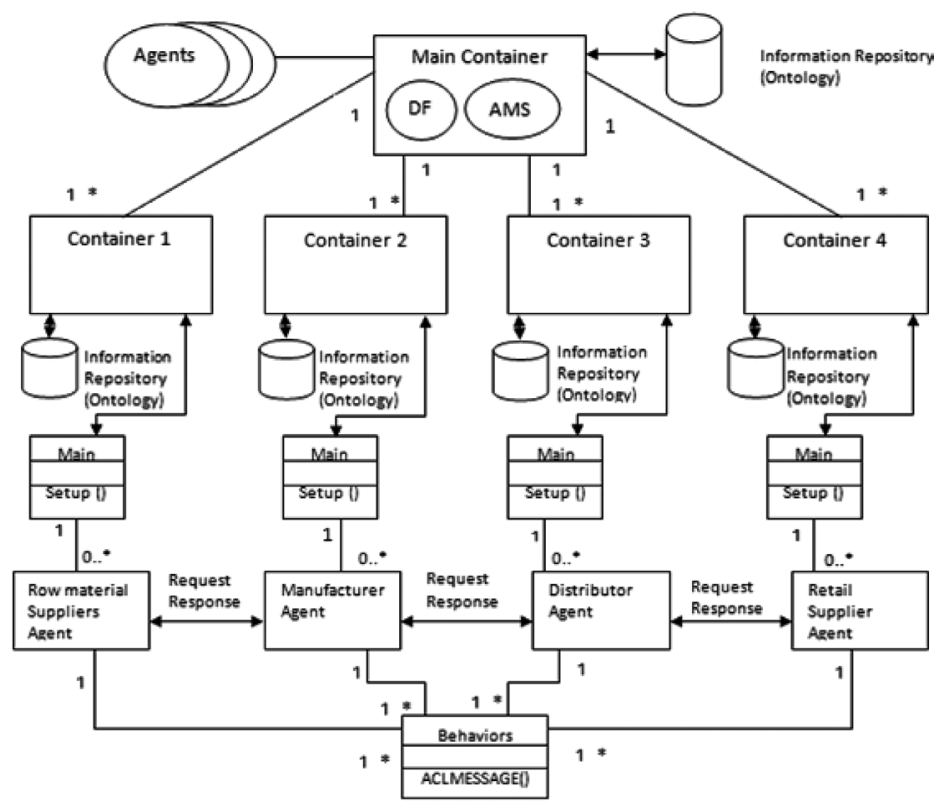

Figure 2: High-level architecture of SCM

It also illustrates that agents are connected to sub-containers to access their personal ontologies while agents are attached to main container also access domain ontology. Agent behaviors are highly coupled with connected ontologies but, main container has authority for agent administration such as transfer agent from one location to another (agent migration), suspend agent whenever it's necessary and terminate an agent. Agent-to-agent communication occurs via message passing and it is included in the diagram as request and response.

\section{OUR PROPOSED METHODOLOGY}

To illustrate the proposed solution, we have included evidence of the resulted outputs. There are two modes of ontologies that have implemented in the system namely personal ontology and domain ontology. However, the knowledge base is physically coded in XML files and MySQL database. In order to generate locally collaborative decisions agents have to access MySQL database and for the global decisions they have to access defined knowledge in XML files. In addition to that the defined ACL message passing mechanism has benefited the successful negotiation in SCM [19]. For implementation purpose Eclipse Luna 4.4.0 has used with JADE [5]. The agents are implemented as separate java classes and their initialization also coded in separate java class.

\section{RESULTS OF THE EFFECTIVE COMMUNICATIONS}

Our aim in this section is to shift the emphasis from design and implementation to practice. It is evident that the results obtained from the communication had with retailer agents and distributer agents are coordinated to deliver and receive products in right time with right quantities.

Figure 3 illustrates decisions have taken by raw material agent and manufacturer agent according to the communication and negotiation procedure. Letter ' $\mathrm{D}$ ' represents the raw material agents while letter ' $S$ ' denotes the manufacture agents. The communication process starts sending offers from manufacturer agent to raw material agent to negotiate the price. If both agents have agreed the prices, 


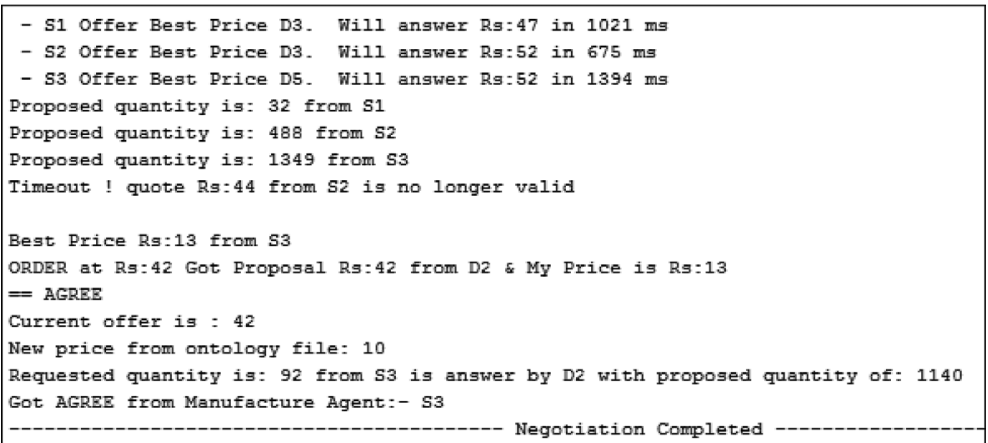

Figure 3: Communication results of raw material and manufacture agents.

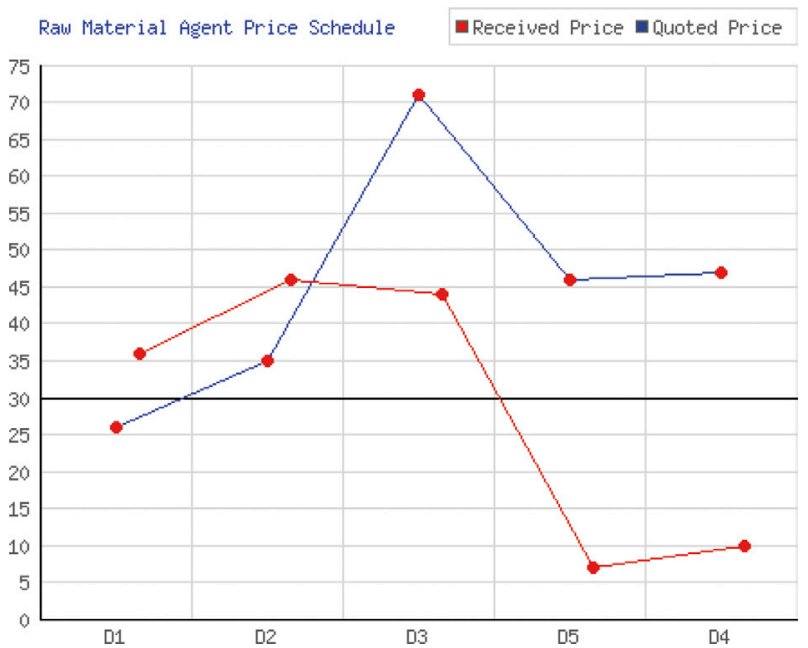

Figure 4: Raw material agents requested and received price schedule.

then they start to negotiate about the supply quantities. When manufacture agents have received the right quantities form according to demand received by retailers the negotiation process will reach to end. Fig. 3 outputs only two categories of agents from SCM domain.

Figure 4 explains that outputs from the end of negotiation process for receiving and offering prices for the certain product category.

According to Fig. 4, 'D1' to 'D5' represents five raw material agents. From the beginning of the communication process D1 received 35 for the quoted 25 and D2 also received 45 for the quoted 35 . However, from D3 to D5 have received lower prices when compared to their quoted prices. This has occurred due to competition among raw materials agents because more raw material agents have joined with the negotiation process. Due to this communication process benefits have received by manufacturer agents because they are able to receive raw materials for competitive prices.

\section{CONCLUSION AND FUTURE WORK}

The literature review and design section have introduced two areas in supply chain mainly information sharing and collaborative planning. We have stated in literature section by outlining some 
industry problems and in what way the concept of multi-agent technology addresses the complexity for solving problems.

After that we have moved into the multi-agent technology as novel approach for the SCM. Our solution described that the features and behaviors of agents and power of multi-agent concept for solving problems, which cannot solve by using conventional SCM applications. We also presented that multi-agent architecture and motivation to use MAS technology by comparing other conventional applications. The system can be applied to forecast trends in supply chain functions and to maximize business objective of an organizations and finally satisfy the end-customer requirements. In fact, the results of the effective communication sections clearly indicate that further negotiation has benefited for the significant change in the SCM which cannot achieve by other conventional technologies. However, we believe that the presented model and multi-agent technology shows potential for the future researchers to eliminate communication complexity in distributed environment.

\section{ACKNOWLEDGMENT}

L.C. M. Perera would like to thank Mrs. Harindra R. Fernando for her great support and encouragement.

\section{REFERENCES}

[1] Moyaux, T., Chaib-draa, B. \& D'Amours, S., supply chain management and multiagent systems: an overview, Springer Abstract, 28, pp. 1-27, 2006. doi: http://dx.doi.org/10.1007/978-3-54033876-5_1

[2] Hernández, J.E., Poler, R., Mula, J., \& de La Fuente, D., A multiagent based-model for the collaborative planning process in decentralized supply chain networks, $3^{\text {rd }}$ International Conference on Industrial Engineering and Industrial Management, Barcelona-Terrassa, September $2^{\text {nd }}-4^{\text {th }}$ 2009, pp. 1012-1020, 2009.

[3] Swaminathan, J.M., Smith, S.F. \& Sadeh, N.M., Modeling supply chain dynamics: a multiagent approach, Decision Sciences, 29(3), pp. 607-632, 1998. doi: http://dx.doi.org/ 10.1111/j.1540-5915.1998.tb01356.x

[4] Kumar, V., \& Srinivasan, S., A review of supply chain management using multi-agent system, International Journal of Computer Science Issues, 7(5), pp. 198-205, 2010.

[5] Um, W., A study of multi-agent based supply chain modeling and management, iBusiness, 2(4), pp. 333-341, 2010.

[6] Min, J.U., Bjornsson, H. Agent based supply chain management automation. Proceedings of the Eighth International Conference on Computing in Civil and Building Engineering (ICCCBE-VIII), pp. 1001-1006, 2000.

[7] Ojha, M., Optimizing supply chain management using gravitational search algorithm and multi agent system. Proceedings of the International Conference on Soft Computing for Problem Solving (SocProS 2011) December 20-22, 2011, pp. 481-491, 2012. doi: http://dx.doi. org/10.1007/978-81-322-0487-9_47

[8] Lau, S.K., \& Li, X., A multi-agent approach towards collaborative supply chain managementIn B. Liu (Eds.), International Conference on Electronic Business. Hong Kong: The Chinese University of Hong Kong, pp. 929-935, 2005.

[9] Fransoo, J.C., \& Wouters, M.J., Measuring the bullwhip effect in the supply chain, Supply Chain Management: An International Journal, 5(2), pp. 78-89, 2000.

[10] Julka, N., Srinivasan, R., \& Karimi, I., Agent-based supply chain management - 1: framework, Computers \& Chemical Engineering, 26(12), pp. 1755-1769, 2002. doi: http://dx.doi. org/10.1016/S0098-1354(02)00150-3

[11] d'Inverno, M., Kinny, D., Luck, M., \& Wooldridge, M., A formal specification of dMARS. Intelligent Agents IV Agent Theories, Architectures, and Languages, Springer, pp. 155-176, 1998. 
[12] Pereira, D., Oliveira, E., Moreira, N., and Sarmento, L., Towards an architecture for emotional BDI agents, in EPIA, vol. 5, pp. 40-47, 2005.

[13] Zhu, X., Agent based modeling for supply chain management: examining the impact of information sharing, Kent State University, 2008.

[14] Carvalho, R. \& Custódio, L., A multiagent systems approach for managing supply-chain problems: new tools and results, Inteligencia Artificial, 9(25), pp. 79-88, 2005.

[15] Popirlan, C.I., Stefănescu, A. \& Stefănescu, L., Multi-agent approach for data analysis in a knowledge-based system for contact centers, World Academy of Science Engineering and Technology, 59, pp. 1126-1131, 2011.

[16] Chopra, A.K., \& Singh, M.P., Elements of a business-level architecture for multiagent systems. Programming Multi-Agent Systems, Springer, pp. 15-30, 2010.

[17] Srinivasan, S., Singh, S.K., and Kumar, V., Multi-agent system based service oriented architecture for supply chain management system, International Journal of Computer Applications, 27(5), pp. 12-16, 2011.

[18] Haitham, A., Applying Electronic Supply Chain Management Using Multi-Agent System A Managerial Perspective, International Arab Journal of e-Technology, 1(3), pp. 106-113, 2010

[19] Fu, Y., Piplani, R., De Souza, R., \& Wu, J., Multi-agent enabled modeling and simulation towards collaborative inventory management in supply chains. Simulation Conference, 2000. Proceedings. Winter, vol. 2, pp. 1763-1771, 2000.

[20] Rady, H.A., Multi-Agent System for Negotiation in a Collaborative Supply Chain Management, International Journal of Video \& Image Processing and Network Security, 11(5), pp. 25-35, 2011.

[21] Chen, Y., Peng, Y., Finin, T., Labrou, Y., Cost, S., Chu, B., Sun, R., \& Willhelm, R., A negotiation-based multi-agent system for supply chain management, Work. Notes Agents, vol. 99, 1999.

[22] Shehory, O.M., Architectural properties of multi-agent systems, Carnegie Mellon University, The Robotics Institute, 1998.

[23] Chen, B., Cheng, H.H., \& Palen, J., Integrating mobile agent technology with multi-agent systems for distributed traffic detection and management systems, Transportation Research Part C: Emerging Technologies, 17(1), pp. 1-10, February 2009.

[24] Wooldridge, M., \& Jennings, N.R., Intelligent Agents: Theory and Practice, 1995. [http:// www.ent.mrt.ac.lk/ ekulasek/it6401/ker.pdf]

[25] Labrou, Y., Finin, T., \& Peng, Y., The current landscape of agent communication languages IEEE Intell. Syst., vol. 14, no. 2, pp. 45-52, 1999. doi: http://dx.doi.org/10.1109/5254.757631

[26] Chopra, A.K., \& Singh, M.P., Agent Communication, MIT Press, 2011, www.csc.ncsu.edu/ faculty/mpsingh/papers/mas/Agent-Communication-chapter.pdf

[27] Foundation for Intelligent Physical Agents, FIPA 97 Specification, Part 2, Agent Communication Language, 1997. [http://www.fipa.org/specs/fipa00018/OC00018.pdf]

[28] Kumar, S., \& Kumar, U., Java agent development framework, International Journal Research, 1(9), pp. 1022-1025, 2014.

[29] Anandampilai, B., Content-based multicasting using JADE, International Journal of Soft Computing, 2(3), pp. 422-425, 2007.

[30] Chen, B., Cheng, H.H., and Palen, J., Integrating mobile agent technology with multi-agent systems for distributed traffic detection and management systems, Transportation Research Part C: Emerging Technologies, 17(1), pp. 1-10, February 2009.

[31] Jennings, N.R., Wooldridge, M., \& Sycara, K., A Roadmap of Agent Research and Development, 1998. [http://users.ecs.soton.ac.uk/nrj/download-files/roadmap.pdf] 